\author{
Supporting Information
}

\title{
Polymer-coated Extracellular Vesicles for Selective Codelivery of Chemotherapeutics and siRNA to \\ Cancer Cells
}

Yong-Yu Jhan ${ }^{a}$, Guillermo Palou Zuniga ${ }^{a}$, Kanwar Abhay Singh ${ }^{a}$, Akhilesh K. Gaharwar $^{a, b}$, Daniel L. Alge $e^{a, b^{*}}$, Corey J. Bishop ${ }^{a^{*}}$

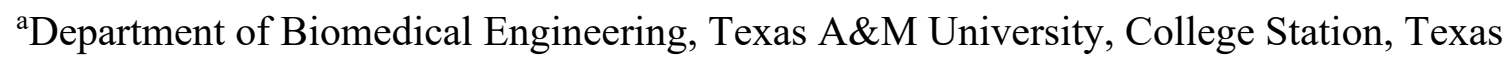
77845-3424, United States

${ }^{b}$ Department of Materials Science and Engineering, Texas A\&M University, College Station, Texas 77845-3424, United States

*Email: dalge@tamu.edu, coreyjbishop@,gmail.com

Number of pages: 6

Number of figures: 6 

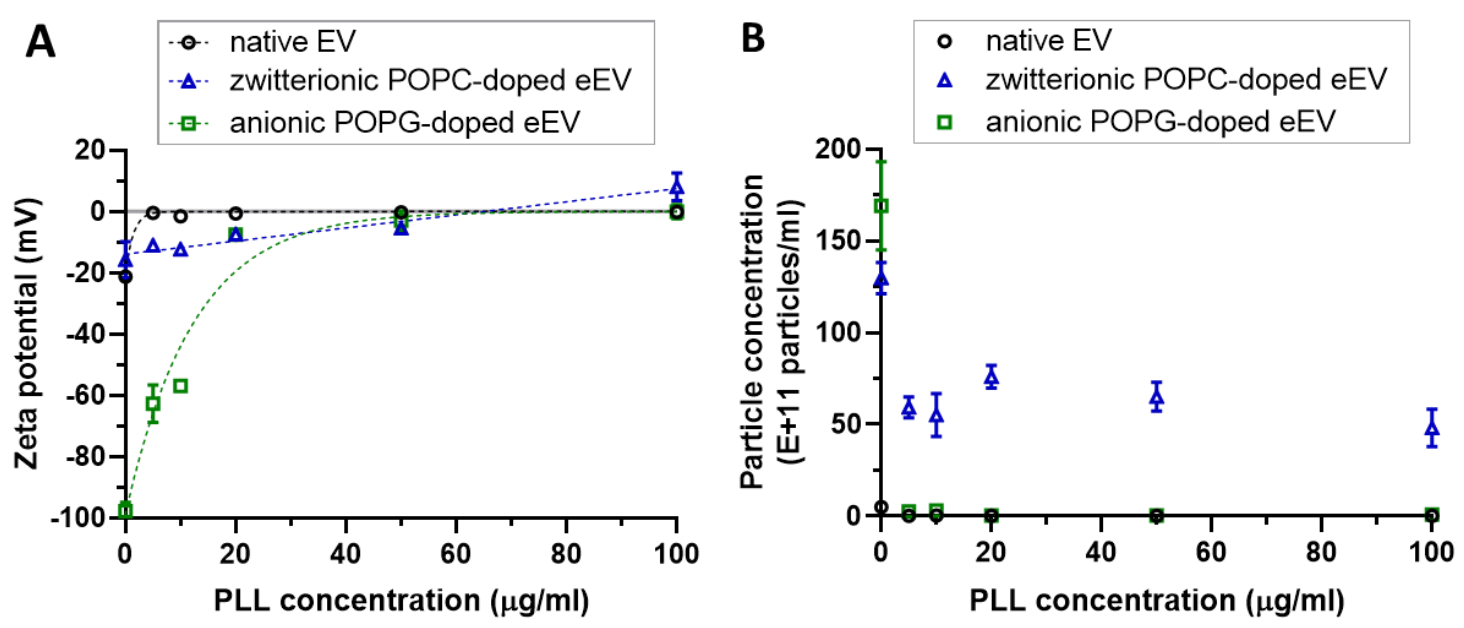

Figure S1. Preliminary tests of PLL deposition on native EV, zwitterionic POPC-doped eEV and anionic POPG-doped eEV via LbL assembly. (A) zeta potential and (B) particle concentration changes with the addition of PLL in the concentration range of $0-100 \mu \mathrm{g} / \mathrm{ml}$.

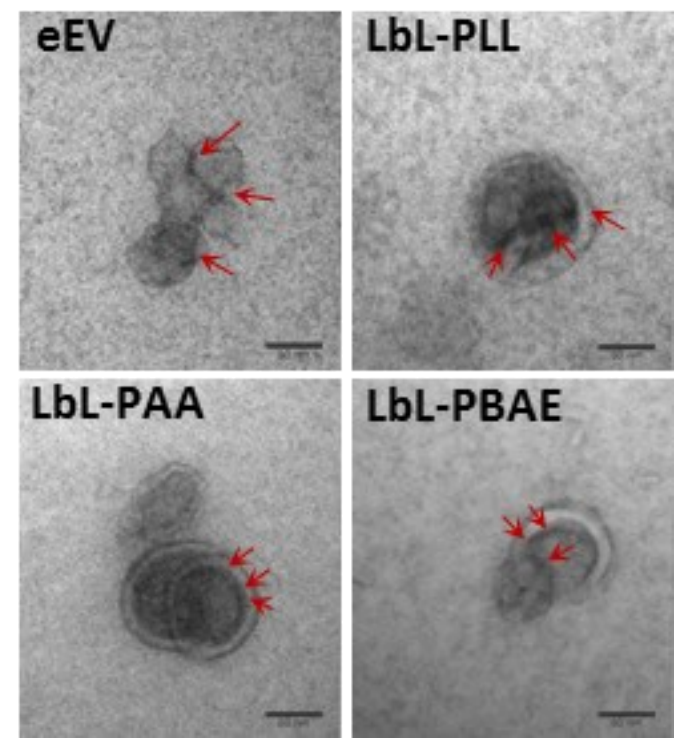

Figure S2. TEM observation of vesicle morphology. Scale bar=50 nm. Black dots indicate the immunogold labeling of CD63 molecules.

Immuno-gold Labelled Transmission Electron Microscopy. LbL-eEVs ( $10^{8}$ particles) aliquots were fixed in 2\% paraformaldehyde and stained on a Formvar/carbon-coated copper grid (Electron Microscopy Sciences) following a protocol in previous literature. ${ }^{1}$ Briefly, $5 \mu 1$ of the sample was placed on a grid for $20 \mathrm{~min}$, followed by a repetitive wash procedure for a total of 3 washes. The grid was then transferred to fresh drops of PBS $/ 50 \mathrm{mM}$ glycine to quench free aldehyde groups. For immunogold staining, the grid was then placed onto a drop of blocking buffer (PBS/5\%BSA) for $10 \mathrm{~min}$. After $10 \mathrm{~min}$, without washing, the grid was transferred to a fresh drop of antibody diluent (PBA/1\%BSA) with the primary antibody CD63 
(4 $\mu \mathrm{g} / \mathrm{ml}$; sc-5275, Santa Cruz) and incubated for $30 \mathrm{~min}$. The grid was then rinsed with PBS and floated on a drop of secondary antibody anti-mouse IgG-gold $10 \mathrm{~nm}$ (G7652, Sigma) for $20 \mathrm{~min}$. Subsequently, the sample grid was stained with uranyl-oxalate solution (Thermo Fisher Scientific), followed by methyl cellulose (Sigma) embedding. The sample grid was then allowed to vacuum dry overnight and observed via TEM (JEOL 1200EX) under $100 \mathrm{kV}$ of energy and $100,000 x$ to $150,000 x$ magnification.

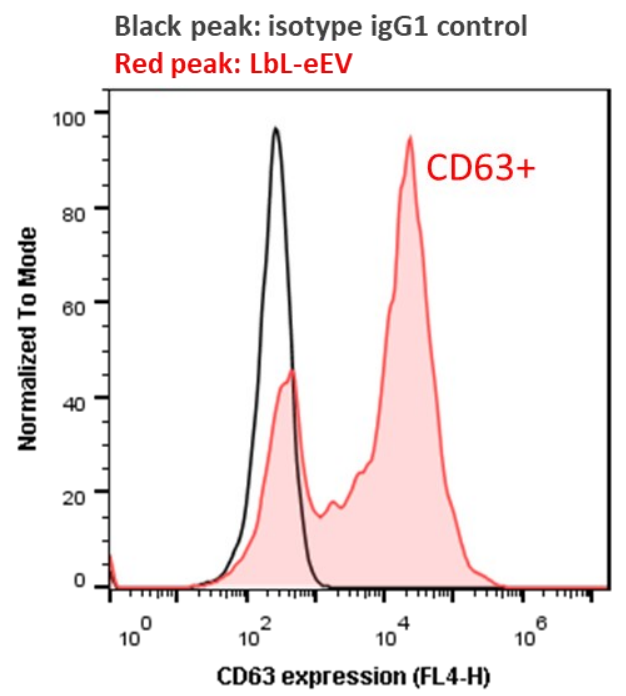

Figure S3. CD63 characterization of LbL-eEV using on bead flow cytometry analysis. LbLeEV was incubated with $4 \mu \mathrm{m}$ sulfate-latex beads, followed by protein blocking and antiCD63-eFluor660 labeling. The histogram reports negative isotype control (black line) and CD63 positive LbL-eEV (red line). 

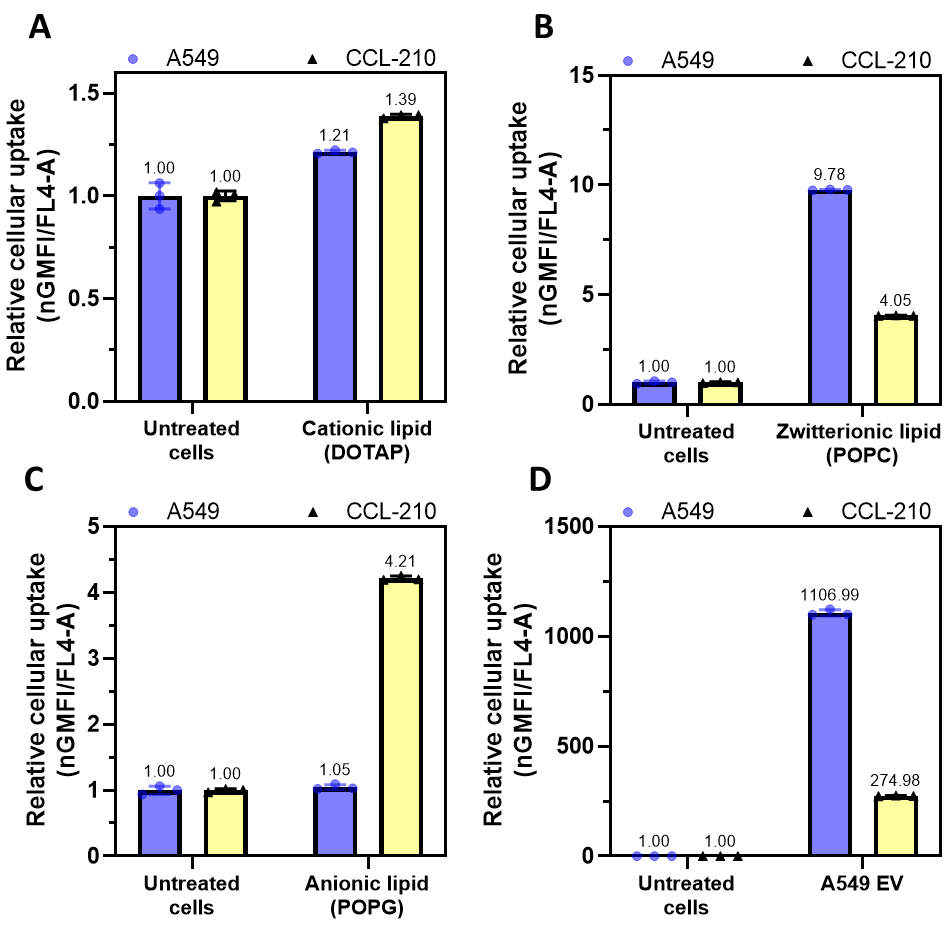

Figure S4. Uptake intensity of A549 and CCL-210 cells after treatment with fluorescently labelled (CellMask DeepRed ${ }^{\circledR}$ dye) liposomes. (A) cationic DOTAP containing liposomes, (B) zwitterionic POPC containing liposomes, (C) anionic POPG containing liposomes, and (D) anionic A549 EVs. Cells were treated for for 2 hours at a concentration of $1.8 \times \mathrm{E} 10$ particles $/ \mathrm{ml}$. The normalized geometric mean fluorescence intensity was calculated by: $n G M F I=$

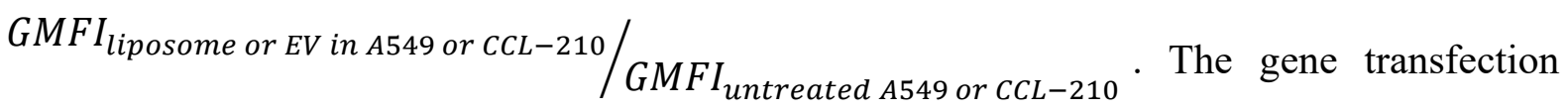
reagent DOTAP liposome and POPG liposome showed a greater amount of vesicle internalization in CCL-210 cells, whereas a higher uptake intensity of POPC liposome was observed in A549 cells. It is worth noting that the preferential uptake of EVs to their parental is likely dose-dependent as discussed in the literature from $\mathrm{Xu}$ et al. ${ }^{2}$ While a 15 -fold greater of A549 EV internalization in A549 cells than CCL-210 cells was reported in the previous studies $\left(10^{11}\right.$ particles $\left./ \mathrm{ml}\right)$, A 4 to 7 -fold greater of A549 EV internalization was observed in A549 cells than CCL-210 cells at $10^{9}-10^{10}$ particles $/ \mathrm{ml}$ 


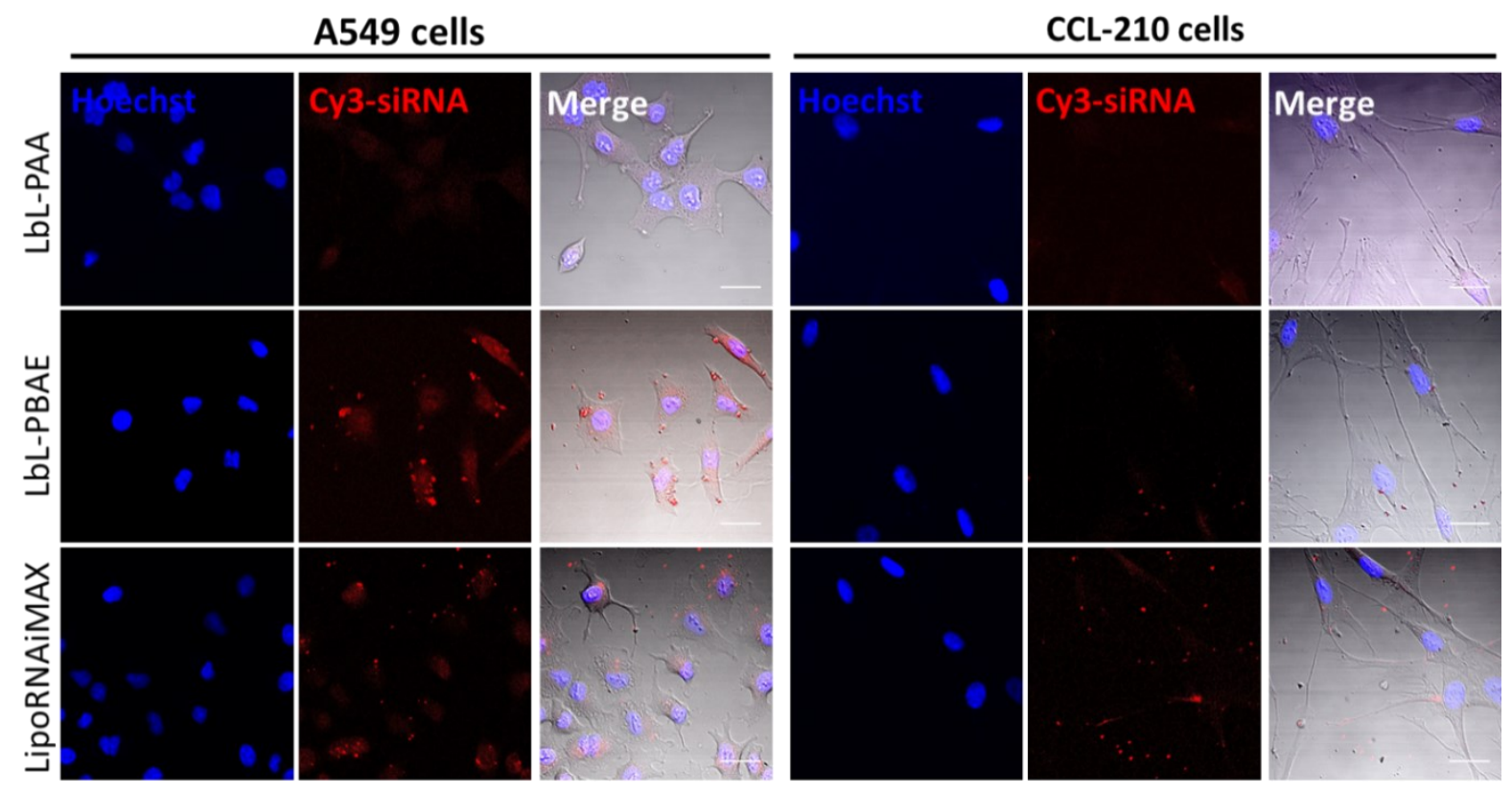

Figure S5. Confocal microscope images of cells treated by different formulations with Cy3-siRNA delivery. Fluorescent images showed Cy3-siRNA in cell cytoplasm. Scale bar: $30 \mu \mathrm{m}$.

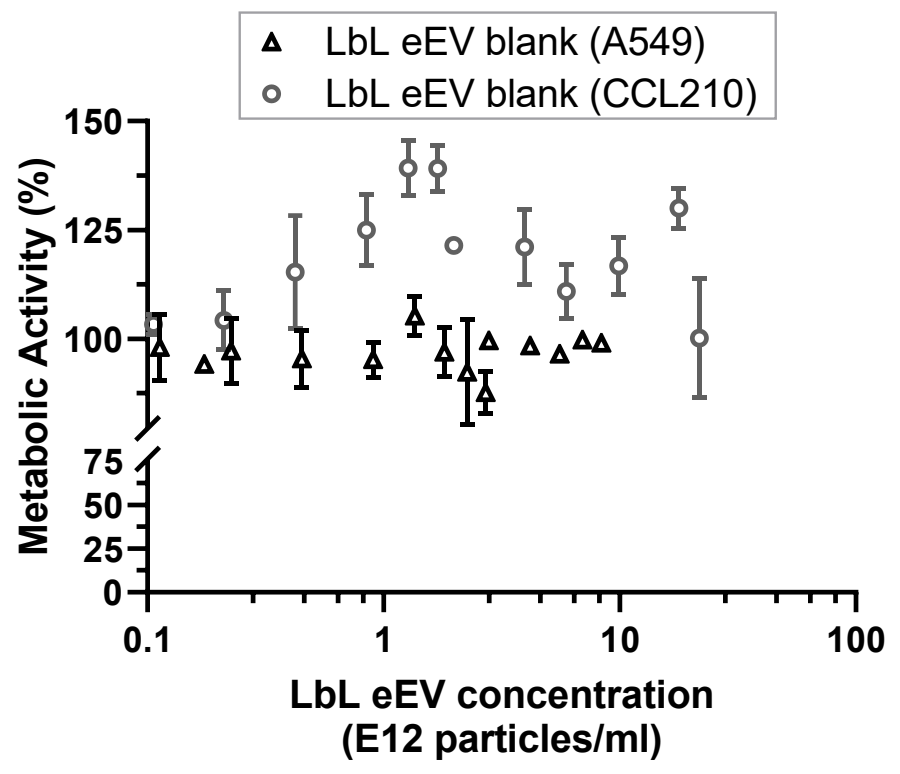

Figure S6. Cell viability of A549 cells and CCL-210 cells incubated with LbL-eEVs at varying concentration for 3 days. The metabolic activity of the cells with different were normalized to untreated A549 or CCL-210 cells.

\section{Reference}

1. Théry C, Amigorena S, Raposo G, et al. Isolation and characterization of exosomes from cell culture supernatants and biological fluids. Current protocols in cell biology 2006: 3.22. 2123.22. 29. 
2. $\mathrm{Xu} \mathrm{L}$, Faruqu FN, Liam-or R, et al. Design of experiment (DoE)-driven in vitro and in vivo uptake studies of exosomes for pancreatic cancer delivery enabled by copper-free click chemistry-based labelling. Journal of Extracellular Vesicles 2020; 9: 1779458. 